\title{
Estrategias de intervención en promoción de la salud
}

\author{
Intervention strategies in health promotion
}

\author{
A. Martínez Rodríguez, E. Martínez Rodríguez, B. Paz Lourido
}

\begin{abstract}
Resumen
La promoción de la salud es un proceso esencial, no exclusivo de las intituciones sanitarias. La promoción de la salud incluye también estrategias políticas, económicas, educativas y sociales. El principal objetivo de este trabajo es resaltar la relevancia de la promoción de la salud, atendiendo a su conceptualización y algunas de las estrategias principales que se pueden desarrollar. Respecto a ello, tienen una relevancia especial las estrategias que no están centradas exclusivamente en el individuo, sino que también toman en consideración el contexto comunitario. Pueden implementarse diferentes estrategias y su nivel de éxito dependerá, en muchos casos, de la interrelación con otras estrategias, la adaptación al contexto, la participación comunitaria y la utilización de recursos de la comunidad.
\end{abstract}

\begin{abstract}
Health promotion is an essential process not only exclusive of health care institutions. Health promotion includes political, economical, educational and social strategies as well. The main aim of this essay is to point out the relevance of health promotion taking into account the concepts involved and the main strategies that can be developed. In this context, estrategies that are not only focused on the individual but more in the community, have a special importance. A variety of strategies can be implemented, and their success will depend in many cases on the level of correlation with other strategies, adaptation to the context, community participation and use of communitary resources.
\end{abstract}

\section{Palabras clave}

Promoción de la salud; Redes comunitarias; Entorno social

Keywords

Health promotion; Community networks; Social environment

\section{Introducción}

En las últimas décadas, la intervención en salud ha vuelto su mirada no sólo a los procesos mórbidos, sino más especificamente a los factores que los determinan y a las consecuencias que en todos los órdenes producen a las personas que los presentan. Los procesos cronicodegenerativos tienen grandes implicaciones en términos de morbilidad y mortalidad, porque, una vez instaurados, estos procesos no suelen ser susceptibles de curación con las herramientas terapéuticas de que se dispone ${ }^{1}$.

Desde la perspectiva histórica de los determinantes de la salud, es conocido el trabajo difundido por el ministro de Sanidad y Asuntos Sociales de Canadá, Marc Lalonde, en cuyo informe ${ }^{2}$ pone de manifiesto la inadecuada visión tradicional de relacionar el nivel de salud con la disponibilidad de médicos y hospitales, y apunta como determinantes para la salud no sólo el sistema sanitario -que además es el que más recursos económicos consume-, sino también el estilo de vida, el ambiente y la biología humana. Dever $^{3}$, en esta misma línea (señala una importancia mayor en la salud del estilo de vida (45\%), de la biología humana (25\%) y del entorno (19\%), respecto a una contribución menor por parte del sistema sanitario (11\%), si bien este último consume el 90,4\% de los recursos invertidos.

Cada problema de salud puede tener determinantes diferentes o con pesos distintos. Por ejemplo, las muertes ocasionadas por accidentes de tráfico están fundamentalmente relacionadas con algunos estilos de vida (por la asunción de riesgos), también en cierta medida con el ambiente (diseño de coches y carreteras) y algo menos con el sistema sanitario (disponibilidad de servicio de emergencias), por lo que 
la contribución de la biología humana es prácticamente nula ${ }^{2}$. Un ejemplo de la importancia del estilo de vida es la incidencia de la muerte por tumor de tráquea/bronquios/pulmón, que se ha duplicado en España desde los años setenta, en relación con el consumo del tabaco 4 .

Por todo ello, resulta más conveniente realizar un esfuerzo de tipo integrador y holístico, dirigido a la promoción de la salud (PS), más que a evitar la enfermedad, y que contemple tanto los aspectos internos (psicológicos y físicos), como los externos a la persona. Estos últimos aspectos están constituidos por factores sociales, económicos, políticos y medioambientales ${ }^{5}$.

La necesidad de un mayor énfasis en aspectos como el ambiente y los estilos de vida no significa que el sistema sanitario sea poco útil o que no pueda contribuir en mayor medida a la salud de la población, ya que todo dependerá del enfoque y las actuaciones que se lleven a cabo. Una referencia histórica hacia una orientación más preventiva y comprensiva del sistema sanitario lo constituyó la Conferencia de Alma-Ata de $1978^{6}$, que supuso un cambio no sólo en la manera de entender la salud, sino en las implicaciones que tiene tanto para el sector sanitario como para los ámbitos social, económico, meioambiental, etc. En España, el cambio de estructura sanitaria, más adaptada al modelo de Alma-Atá se encuentra en la Reforma de la Atención primaria ${ }^{7 \text { and } 8}$ (Real Decreto 137/1984 de 11 de enero; BOE 1 febrero y Estructuras básicas de salud. Ley 14/1986 de 25 de abril; BOE 29 de abril) y la puesta en marcha del Programa de Actividades Preventivas y de Promoción de la Salud en Atención Primaria por parte de los equipos de atención primaria. En el ámbito internacional y en el mismo sentido, se identificó la reorientación de los servicios de salud en la Carta de Otawa como una de las 5 áreas de acción prioritaria ${ }^{9}$. Sin embargo, en la actualidad, el sistema sanitario español sigue estando más orientado clínicamente que preventivamente ${ }^{4}$. Por eso, se hace más importante un esfuerzo de otros sectores y de la población, que incidan en la obtención de una salud mayor para la comunidad.

La intervención comunitaria parte de la necesidad de involucrar a la comunidad en la búsqueda de soluciones para abordar los problemas que le afectan. De poco sirve planificar intervenciones preventivas si no se diseñan desde las necesidades de la comunidad, con la participación de ésta en todo el proceso. La comunidad es la que conoce la realidad en la que vive, la que elabora un determinado discurso sobre la salud y la enfermedad, la que define con qué recursos cuenta, y la que en último término debe decidir hacia dónde quiere encaminarse ${ }^{10}$.

Por este motivo, dotar de un papel activo a la comunidad y fomentar su participación en el quehacer preventivo es potenciar su capacidad de decisión y de control respecto a la realidad que vive. También constituye una forma de fortalecer a la propia comunidad, como espacio preventivo y de desarrollo individual y colectivo ${ }^{10}$.

\section{Objetivo}

El objetivo de este artículo es proponer una concepción de intervención en prevención a través de una perspectiva holística e integradora, orientada desde el eje de la salud y no desde la enfermedad. Se pretende traspasar la visión más restrictiva de la prevención de la enfermedad, en dirección a la PS, considerando protagonistas y responsables de la actuación no sólo a la persona o al grupo, sino también a la comunidad y el contexto social. Las estrategias de intervención serán, por tanto, múltiples, complementarias entre sí y buscarán actuar tanto en el ámbito del individuo, como en el entorno.

\section{Campo conceptual de la prevención y promoción de la salud}

La prevención de los problemas de salud se sitúa a 3 niveles distintos e interrelacionados. Son:

1. La prevención primaria, que actúa en las causas de los problemas de salud ${ }^{11}$, con la disminución de los factores de riesgo o el aumento de los factores de protección. Su objetivo es disminuir la incidencia de la enfermedad ${ }^{1}$.

2. La prevención secundaria, que se centra en la detección temprana de los problemas de salud ${ }^{11}$ y en detener su progreso. Persigue la reducción de la prevalencia de la enfermedad ${ }^{1}$.

3. La prevención terciaria, que se dirige al tratamiento y la rehabilitación de una persona con una enfermedad o proceso mórbido ya establecido, lentificando o anulando su progresión, y con ello la aparición o el agravamiento de complicaciones e invalidez ${ }^{1}$.

Generalmente, la prevención se asocia a las acciones que emanan del contexto clínico para evitar o gestionar factores de riesgo o comportamientos de riesgo. Pero un enfoque más amplio (la comunidad en lugar de la persona) y positivo (centrado en la salud y no en la enfermedad) respecto al preventivo es el de la PS. La Organización Mundial de la Salud (OMS) ha definido este término como el proceso que 
capacita a los individuos y a la comunidad para aumentar su control en los determinantes de salud y, por lo tanto, para mejorarla ${ }^{12}$. Green y Kreuter, citados por Van den Borne ${ }^{13}$, definen la PS como cualquier combinación de soporte educacional, político, legislativo y organizativo para las acciones y las condiciones de vida que conducen a la salud de los individuos, los grupos o las comunidades.

Por tanto, la PS constituye un proceso político y social global que abarca no solamente las acciones dirigidas directamente a fortalecer las habilidades y las capacidades de los individuos, sino también las dirigidas a modificar las condiciones sociales, ambientales y económicas, con el fin de mitigar su impacto en la salud pública e individual ${ }^{12}$. De esta manera, las estrategias de PS se dirigen tanto a los individuos como a la comunidad, desde un punto de vista holístico y transformador, entendiendo la intervención comunitaria como un conjunto de acciones destinadas a promover el desarrollo de una comunidad, a través de la participación activa de ésta en la transformación de su propia realidad. De manera más concreta podemos conceptualizar la PS en el ámbito comunitario, entendiendo la comunidad de 3 maneras distintas ${ }^{10}$.

1. La comunidad como objeto de intervención, es decir, la posibilidad de incidir en los colectivos y las personas que la forman, buscando potenciar los factores de protección y disminuir los de riesgo.

2. La comunidad como sujeto de intervención, a través de la capacitación de colectivos y personas que actúen como mediadores en prevención y como interlocutores entre la comunidad y las instituciones.

3. La comunidad como espacio donde se desarrollan actitudes, discursos y respuestas sociales ante los distintos fenómenos relacionados con la salud, en los que hay que intervenir.

Siguiendo este enfoque, el sistema sanitario debe hacer un esfuerzo por alejarse de una orientación individualista y acercarse a una perspectiva comunitaria, incluyéndola no sólo como objeto, sino también como sujeto y espacio. En consecuencia, la filosofía de intervención propuesta desde este artículo es la de la PS, considerada desde una perspectiva más amplia que la prevención de la enfermedad y, por tanto, muy útil para incidir en el cambio de actitudes y comportamientos en salud. A continuación, se presenta una serie de estrategias que pueden ponerse en marcha desde el ámbito sanitario para, por un lado, considerar el marco más ampliado y cercano a la realidad y, por el otro, favorecer la participación comunitaria.

\section{Estrategias de promoción de la salud}

Como ya se ha señalado, el enfoque propuesto se dirige a la persona y la comunidad inmersas en su tejido social, económico, político y cultural. Cuando la intervención se realice en personas determinadas, no se las considerará sólo como objeto de prevención, sino como posibles agentes preventivos y de PS. Por tanto, la actuación se programará teniendo en cuenta su posible influencia en otras personas, ya sea a nivel interpersonal o social, en el caso de personas relevantes en la comunidad, que puedan actuar de mediadores sociales.

Fundamentalmente, se hablará de las estrategias de PS siguientes: $a$ ) estrategia de influencia; $b$ ) estrategia de desarrollo de competencias; $c$ ) estrategia de modificación del medio, y $d$ ) estrategia de control. Esta división en 4 áreas de intervención permite incidir desde diversos ámbitos y estructurar una línea de cambio global, con el desarrollo de factores de protección y la disminución de los factores de riesgo tanto internos, como externos a la persona. Permite, además, abordar la complejidad y la multiplicidad de las problemáticas relacionadas con la salud de las personas, porque recuerda la necesidad de complementar las diversas acciones, ya que es difícil que intervenciones aisladas desde cualquiera de las estrategias consigan el cambio.

\section{Estrategia de influencia}

Esta estrategia se basa en el modelo de cambio de actitudes, cuyo argumento se sustenta en que predisponer positivamente hacia la acción, la facilita. Se apoya en la información, la sensibilización y el cambio de valores. Se puede dirigir a una persona o grupo, o a la comunidad, y se obtienen mejores resultados cuando las personas socialmente relevantes para el grupo o persona objeto de intervención son las que motivan hacia el cambio. Es la estrategia más utilizada en prevención; no obstante, de forma puntual y aislada no obtiene grandes resultados ${ }^{14}$.

La comunicación en esta estrategia debe ser persuasiva para que sea efectiva, y esto se consigue teniendo en cuenta todas las variables que afectan al proceso comunicativo ${ }^{15}$, a saber: fuente, mensaje o contenido, destinatario/a/s y medio de transmisión del mensaje. 
En cuanto a la fuente, se sabe que condiciona la atención del/de la receptor/a, así como su conformidad con el mensaje. Si la fuente resulta atractiva, aumenta la atención prestada y la disposición a adoptar su perspectiva. En la percepción de veracidad del mensaje, influye el prestigio y la competencia de la fuente, al igual que su credibilidad, que es mayor cuando el mensaje transmitido está en contra de su propio interés ${ }^{15}$. Además, si la fuente se percibe como semejante, permite la identificación con el modelo, sobre todo cuando faltan conocimientos y experiencia en el tema, o cuando se trata de aspectos que no pueden contrastarse de forma objetiva ${ }^{16}$.

En relación con los propios mensajes, será más fácil entenderlos y recordarlos si se ofrecen repetidamente a través del mayor número posible de fuentes y se presentan en términos concretos y fáciles de seguir. Se debe huir de la abstracción excesiva y facilitar la comprensión con ayudas didácticas y con ejemplos, en lugar de con cifras y porcentajes ${ }^{17}$. Han de resultar claros, y emplear una argumentación que se corresponda con la visión del mundo que tengan los/las receptores/as y un vocabulario adaptado a ellos/as ${ }^{18}$. Además, deben ser veraces y completos, así como proporcionar todas las claves necesarias para la comprensión del problema y evitar el miedo y la amenaza, ya que ante la fuerte carga emocional negativa, la persona pondrá en juego mecanismos de negación que dificultarán la actuación racional y la efectividad a medio y largo plazo. Resulta más adecuado el mensaje positivo, así como enfatizar las consecuencias probables y deseables del comportamiento ${ }^{19}$.

En cuanto a las circunstancias asociadas a los/as destinatario/a/s, hay que tener en cuenta aspectos como la edad, la inteligencia y la autoestima, o factores muy variables, como la posición ante el mensaje y el grado de implicación.

La edad condiciona tanto los intereses, como las posibilidades de comprensión y acción de las personas, mientras que la inteligencia y, especialmente, la autoestima pueden influir de forma diversa en función de las demás variables. Una autoestima baja puede proporcionar una facilidad mayor en el convencimiento de la idoneidad del cambio propuesto, pero primero la persona tiene que haber atendido al mensaje, lo que es menos probable por el bajo interés por el mundo que le rodea ${ }^{15}$.

El grado de discrepancia con la posición defendida generalmente influye prestando más atención a los mensajes que concuerdan con nuestras ideas y teniendo más en cuenta lo que concuerda con nuestras posiciones ${ }^{15}$, aunque el mayor cambio de actitudes se produce con una discrepancia moderada ${ }^{20}$.

Por otra parte, un grado alto de implicación hará que los individuos se esfuercen más en analizar el mensaje y sus consecuencias, y observar sus consistencias y sus posibles errores. Un grado bajo de implicación llevará a la persona a fijarse en aspectos más superficiales, como por ejemplo la credibilidad de la fuente, para decidir su posición frente al mensaje ${ }^{15}$.

El medio de transmisión del mensaje (o también denominado vehículo) más influyente es el personal $^{14}$, si bien los medios de comunicación ejercen, asimismo, un peso persuasivo notable. En cualquier caso, es más eficaz si es consistente y diverso, que promueva la discusión y el debate. También, si se presenta en un material atractivo que se conserve y use, porque además tenga otra utilidad. Lo importante es que permita al receptor manipularlo, reflexionar y asimilar la información a su ritmo, según sus necesidades y capacidad.

Con la atención a todas estas variables, se pretende que la persona o la comunidad se implique lo máximo posible para que pueda realizarse un análisis lo más concienzudo y completo posible, y extraer sus propios objetivos.

\section{Estrategia de desarrollo de competencias}

Con esta estrategia, se trata de suministrar las herramientas necesarias para poder efectuar el cambio de comportamiento que mantenga o mejore la salud. Se puede y debe contar con las personas que estén más cercanas, sean más influyentes y puedan participar o ayudar en la adquisición o el perfeccionamiento de las habilidades en cuestión. No basta en conocer y tener una opinión positiva frente a determinados comportamientos saludables, sino que deben llevarse finalmente a cabo, cuestión que parece estar positivamente relacionada con la autoestima alta, la autonomía y la capacidad para la toma de decisiones $^{14}$.

Este último punto es el que trataremos de considerar en nuestro trabajo diario, incluidas estrategias para mantener o incrementar la autoestima y la sensación de control, mientras estamos proporcionando las herramientas pertinentes para que pueda llevar a cabo el cambio decidido.

Con este fin, se emplea una comunicación que muestre nuestra consideración e interés, así como animar y ayudar en los momentos en que las personas se sientan con dificultades ${ }^{21}$.

En ocasiones, las personas se bloquean con pensamientos del tipo "yo no sé", "yo no puedo" o "yo no valgo". Hay que intentar cambiar estas percepciones hacia otras más positivas y facilitadoras del cambio, como "puedo aprender". También es conveniente no hacer comparaciones entre personas que resulten negativas para una/s de ellas. 
Para que la persona continúe motivada en la dirección del cambio, ha de gozar de experiencias positivas que le proporcionarán más sensación de control. Es importante ayudar a fragmentar las metas, de modo que se vaya de lo más fácil a lo más complicado, o pedir participación inicial en situaciones ya conocidas. Como ejemplo más simple, en un programa preventivo de ejercicios, el/la fisioterapeuta puede partir de los que resulten motormente menos complejos, y que no generen sensación dolorosa, o que las personas conozcan por haberlos realizado con anterioridad o ser similares a otros aprendidos. El/la fisioterapeuta también puede ayudarse de refuerzos cuando las personas se esfuercen o vayan consiguiendo realizarlos más correctamente.

\section{Estrategia de modificación del medio}

Además de que la persona esté informada, motivada y disponga de las habilidades necesarias para llevar a cabo determinada acción, se requiere que el entorno la facilite o, al menos, no la impida. La estrategia de modificación del medio pretende mejorar los espacios donde se desenvuelven los individuos, así como disminuir los factores de estrés en estos espacios y las consecuencias negativas de los riesgos para las personas o la sociedad. También persigue facilitar la realización de comportamientos saludables y dificultar la realización de conductas no saludables. Un ejemplo de como el medio puede influir en las conductas saludables puede ser el de una persona hipertensa que conoce los beneficios del ejercicio aeróbico y está motivada para hacerlo, además de saber graduar la actividad, hidratarse correctamente, controlar su presión arterial, seleccionar el calzado y el terreno más adecuado, etc. Sin embargo, puede que finalmente no lo lleve a cabo, porque vive al pie de una carretera peligrosa que ha de cruzar. En cambio, tener un parque cercano puede ayudar a decidirse o facilitar el hecho de ir a caminar a alguien que no está tan motivado.

Se debe trabajar directamente e indirectamente, y apoyar a las personas y la comunidad en la petición de entornos más saludables, que disminuyan factores de riesgo que no se pueden controlar individualmente (p. ej., contaminación) y que inviten a la acción saludable (creación de parques, piscinas, etc.). Otra posibilidad es la reutilización de espacios ya existentes para otras actividades (p. ej., abrir las escuelas por las tardes para actividades del barrio). En este sentido, la propia OMS inició la creación de una red de ciudades saludables ${ }^{22}$, que en Europa se encuentra ya en su cuarta fase. España participa formalmente en ella desde el año 1988, con la intervención del Ministerio de Salud y Consumo, redes regionales de ciudades saludables y otras entidades comprometidas en la promoción y la protección de la salud.

Además de la facilitación de conductas saludables y la disminución de los aspectos estresantes del ambiente, la modificación del medio puede dirigirse a los comportamientos perjudiciales para la salud. En este caso, el ambiente puede bien generar dificultades para la realización de éstos, bien minimizar sus efectos adversos. Como ejemplo de la primera posibilidad, para la compra de bebidas alcohólicas o tabaco, la obligación de pedirlas al dependiente, en vez de poder escogerlas libremente a través de máquinas dispensadoras o en un autoservicio, limita el consumo abusivo. Este sistema se ha establecido en el caso del tabaco en España, de modo que hay que solicitar al/a la dependiente/a que activen la maquina $^{23}$. En el segundo caso, cuando se mantiene el comportamiento de riesgo, las consecuencias negativas disminuyen si se dispone de elementos que las mitiguen, como por ejemplo, en el caso del tabaco, extractores de humo.

\section{Estrategia de control}

Esta estrategia se basa en el establecimiento de un control externo a la persona o a la comunidad que, a corto plazo, restrinja los beneficios de la asunción de conductas no saludables y/o introduzca costes, reconocidos y relacionados con el comportamiento a evitar. Deriva del modelo de modificación de la conducta, y es a menudo criticada por considerarse que establecer un control externo es limitar la libertad de la persona. Pero el control externo es necesario para aprender que nuestras actuaciones tienen límites y consecuencias, así como conseguir reconocerlos y, por tanto, aprender a autocontrolarnos y a tomar decisiones conscientes y responsables de sus consecuencias. Así se podrá decidir entre evitar o asumir las consecuencias negativas a largo plazo, evitar la frustración de las consecuencias negativas no anticipadas y perseguir consecuencias positivas. Todo esto nos permite actuar con libertad y sensación de control ${ }^{14}$.

Las medidas legislativas y judiciales infligen costes externos por realizar conductas que pueden acarrear costes sociales o riesgos para la salud pública a corto y a largo plazo. Estas medidas se añaden a las posibles consecuencias negativas de los comportamientos no saludables; por lo tanto, tienen que percibirse como muy probables, ya que si se perciben como eventuales no disuaden a las personas más que las consecuencias negativas en sí y, por tanto, no son preventivas. Un ejemplo en relación con el 
tráfico puede ilustrar estas afirmaciones. No es tan importante que la multa por exceso de velocidad sea alta, si se aplica pocas veces y, por tanto, se percibe como improbable, ya que el coste, aunque alto, no lo es más que el de un accidente. En ambos casos, las personas piensan que a ellas no les va a pasar. Las medidas graduales, pero aplicadas con rigidez, son mucho más efectivas que las medidas muy duras, pero que en la práctica no se aplican. Un ejemplo es el actual carné de conducir español por puntos, donde al infringir determinadas normas de tráfico se van perdiendo puntos (además de poder ser multado). Estos castigos pueden tener un carácter más social o personal, sin que tenga que estar siempre relacionado con normativa de tipo legal.

Las medidas derivadas de la estrategia de control no enseñan cómo actuar, ya que sólo ponen límites a las actuaciones no deseadas. Por eso, para que sean efectivas deben utilizarse siempre como complemento de otras estrategias. Algo muy básico para su buen funcionamiento es que se esté informado de qué es lo que se espera de uno y que pueda efectivamente llevarse a cabo. Si la persona no sabe qué es mejor hacer para su salud, ni las consecuencias que se derivan de los comportamientos que adopte, parece poco razonable imponer un castigo. Un ejemplo de estrategias combinadas lo constituyó uno de la campaña RENFE (Red Nacional de los Ferrocarriles Españoles) en contra del viaje en tren sin billete; la estrategia de control de la multa se combinó con una estrategia de influencia, en la que se informaba de la campaña y del porqué de ella, de los beneficios y los costes para todos de la trasgresión, dando un sentido a las multas. Además, se empleó una estrategia de modificación del medio, con el aumento de los controles y los cierres con control de billetes a las salidas, mientras que se incrementaban las máquinas para sacar el billete y se hacía hincapié en los sistemas de abono para reducir costes para el usuario.

Otro aspecto importante en PS es que las medidas de control se hayan consensuado previamente con la/s persona/s objeto de la intervención, de modo que se busque la predisposición y la aceptación de los comportamientos y las consecuencias, e incluso la propia autoadministración de éstas (según las dificultades percibidas). En el caso de la comunidad, ésta debe haber podido participar en las decisiones correspondientes a través de mecanismos formales e informales.

\section{Consideraciones finales}

Como los determinantes de salud son múltiples, en los párrafos anteriores se han presentado algunas de las estrategias más relevantes dirigidas a incidir en estos determinantes. La excepción la constituye el determinante biológico, pues éste cae fuera de nuestras posibilidades.

Estas estrategias deben cumplir una serie de premisas básicas en cuanto a su diseño e implementación. En primer lugar, resulta fundamental que las estrategias estén contextualizadas, adaptadas a los objetivos y a la población/persona a la que se dirige. En segundo lugar, deben responder a la globalidad y a la complejidad, lo que se consigue aplicando varias estrategias, de forma que se complementen o refuercen entre sí. De esta manera, se obtiene un alcance mayor, que comprende las interrelaciones que pudieran haber para lograr un efecto de más entidad y amplitud.

Por último, las estrategias también deben dirigirse a la movilización de los recursos y las redes comunitarias del entorno, con el fortalecimiento de las redes de apoyo social informal. De esta manera, se facilitará que los cambios realizados se puedan mantener en el tiempo.

\section{Bibliografía}

1 Martín A, Gené J, Subías P. Actividades preventivas y de promoción de la salud. En: Martín Zurro A, Cano Pérez JF, editores. Atención Primaria. Conceptos, organización y práctica clínica, 5. a ed. Madrid: Elsevier España; 2003.

2 Minister of Supply and Services Canada 1981. A New Perspective on the Health of Canadians. A working document. [Consultado 3/14/07] Disponible en: http://www.phac-aspc.gc.ca/ph-sp/phdd/pdf/perspective.pdf.

3 G. Dever An epidemiological model for heatlh analysis Soc Ind Res, 2 (1976), p. 465

4 A. Durán, J.L. Lara, M. Van Waveren Spain: Health System Review Health Systems in Transition, 8 (2006), pp. 1208 Disponible en http://www.euro.who.int/Document/E89491.pdf View Record in Scopus

5 Who Centre for Health Development. Ageing and Health Technical Report. Volume 5. A glossary of terms for community health care and services for older persons. WHO/HPR/HEP/98.1.Geneva: World Health Organization; 2004.

6 Atención Primaria de Salud. Informe de la Conferencia Internacional sobre Atención Primaria de Salud. Alma Ata, URSS, 6-12 de septiembre de 1978. Ginebra: OMS; 1978.

7 Real Decreto 137/1984 de 11 de enero, de Estructuras básicas de salud. BOE 1 de febrero.

8 Ley 14/1986 de 25 de abril, General de Salud. BOE 29.

9 Ottawa Charter for Health Promotion WHO/HPR/HEP/95.1 World Health Organization, Geneva (1986) 
10 E. Martínez, A. Mendoza Prevención de los consumos de drogas en el ámbito comunitario E. Alemany, C. Alonso, G. Lerma, E. Martínez, S. Méndez, A. Mendoza (Eds.), et al., La comunidad y la prevención de los consumos de drogas, FAD y Gobierno de la Provincia de Buenos Aires, Madrid (1999)

11 A. Rochon Educación para la salud. Guía práctica para realizar un proyecto Masson, Barcelona (1992)

12 Health Promotion Glossary WHO/HPR/HEP/98.1 World Health Organization, Geneva (1998) 13 H.W. Van den Borne The patient from receiver of information to informed decision-maker Patient Educ Couns, 34 (1998), pp. 89-102

14 C. Arenas, E. Martínez, E. Vela, M. Pérez-Lozao Trabajo en Red y Gestión de Riesgos en el Ocio Juvenil. Formato CD Grupo GID y Plan Nacional sobre Drogas, Madrid (2004)

15 Moya M. Persuasión y cambio de actitudes. En: Morales JF, Huici C, coordinadores. Psicología Social. Madrid: McGraw-Hill/Interamericana de España; 2000.

16 G.R. Goethals, R.E. Nelson Similarity in the influence process: the belief-value distinction J Pers Soc Psychol, 25 (1973), pp. 117-122

17 S.E. Taylor, S.C. Thompson Stalking the elusive "vividness" effect Psychol Rev, 89 (1982), pp. 155-181

18 M.A. Modolo Educazione sanitaria Edizioni Rosini, Florencia (1974)

19 R.E. Petty, D.T. Wegener Thoughts systems, argument quality, and persuasion R.S. Wyer, T.K. Srull (Eds.), Advances in social cognition, Vol. 1, Erlbaum, Hillsdale, NJ (1991), pp. 147-161

20 D. O'Keefe Persuasion: theory and research Sage, Newbury Park (1990)

21 M. Costa, E. López Educación para la salud. Una estrategia para cambiar los estilos de vida Pirámide, Madrid (1998)

22 Lafond LJ, Heritage Z, Farrington JL, Tsouros A. National healthy cities networks. A powerful force for health and sustainable development in Europe. World Health Organization, 2003. Disponible en: http://www.euro.who.int/document/e82653.pdf.

23 Ley 28/2005, de 26 diciembre de Medidas sanitarias frente al tabaquismo y reguladora de la venta, el suministro, el consumo y la publicidad de los productos del tabaco (BOE 27 diciembre 2005) 